\title{
Some Properties of Cartesian Product Graphs of Cayley Graphs with Arithmetic Graphs
}

\author{
S. Uma Maheswari \\ Lecturer \\ Department of Mathematics \\ JMJ College for Women \\ Tenali, AP, India
}

\author{
B. Maheswari \\ Professor \\ Department of Applied Mathematics \\ SP Women's University \\ Tirupati, AP, India
}

\begin{abstract}
Nathanson was the pioneer in introducing the concepts of Number Theory, particularly, the "Theory of Congruences" in Graph Theory. Thus he paved the way for the emergence of a new class of graphs, namely "Arithmetic Graphs". Cayley graphs are another class of graphs associated with the elements of a group. If this group is associated with some arithmetic function then the Cayley graph becomes an Arithmetic graph.
\end{abstract}

Graph product is a fundamental tool with rich applications in both graph theory and theoretical computer science. The extensive literature on products that has evolved over the years presents a wealth of profound and beautiful results.

In this paper, results related to some properties of Cartesian product graphs of Euler totient Cayley graphs with Arithmetic $V_{n}$ graphs are determined.

\section{Keywords}

Euler totient Cayley graph, Arithmetic $V_{n}$ graph, Cartesian product graph.

AMS (MOS) Subject Classification: 6905c

\section{INTRODUCTION}

\section{EULER TOTIENT CAYLEY GRAPH $G\left(Z_{n}, \varphi\right)$ AND ITS} PROPERTIES

Madhavi [1] introduced the concept of Euler totient Cayley graphs and studied some of its properties. She gave methods of enumeration of disjoint Hamilton cycles and triangles in these graphs. Sujatha [2] studied some cyclic structures of Euler totient Cayley graphs.

For any positive integer $n$, let $Z_{n}=\{0,1,2, \ldots . n-1\}$ be the residue classes modulo $n$. Then $\left(Z_{n}, \oplus\right)$, where, $\oplus$ is addition modulo $n$, is an abelian group of order $n$. The number of positive integers less than $\mathrm{n}$ and relatively prime to $n$ is denoted by $\varphi(n)$ and is called Euler totient function. Let $S$ denote the set of all positive integers less than $n$ and relatively prime to $n$. That is $S=\{r / 1 \leq r<$ $n$ and $\operatorname{GCD}(r, n)=1\}$. Then $|S|=\varphi(n)$.

Now define Euler totient Cayley graph as follows.

For each positive integer $n$, let $Z_{n}$ be the additive group of integers modulo $n$ and let $S$ be the set of all integers less than $n$ and relatively prime to $n$. The Euler totient Cayley graph $G\left(Z_{n}, \varphi\right)$ is defined as the graph whose vertex set $V$ is given by $Z_{n}=\{0,1,2, \ldots . n-1\}$ and the edge set is $E=\{(x, y) / x-y \in S$ or $y-x \in S\}$.

Clearly as proved by Madhavi, the Euler totient Cayley graph $G\left(Z_{n}, \varphi\right)$ is
1. a connected, simple and undirected graph,

2. $\varphi(n)$ - regular and has $\frac{n . \varphi(n)}{2}$ edges,

3. Hamiltonian,

4. Eulerian for $n \geq 3$,

5. bipartite if $n$ is even and complete graph if $n$ is a prime.

\section{ARITHMETIC $V_{n}$ GRAPH}

Vasumathi [3] introduced the concept of Arithmetic $V_{n}$ graphs and studied some of its properties.

Let $n$ be a positive integer such that $n=p_{1}^{\alpha_{1}} p_{2}^{\alpha_{2}} \ldots \ldots . . p_{k}^{\alpha_{k}}$. Then the Arithmetic $V_{n}$ graph is defined as the graph whose vertex set consists of the divisors of $n$ and two vertices $u, v$ are adjacent in $V_{n}$ graph if and only if $\operatorname{GCD}(u, v)=p_{i}$, for some prime divisor $p_{i}$ of $n$.

In this graph the vertex 1 becomes an isolated vertex. Hence consider the Arithmetic graph $V_{n}$ without vertex 1 because the contribution of this isolated vertex is nothing when the properties of these graphs and enumeration of some domination parameters are studied.

Clearly, $V_{n}$ graph is a connected graph. Because if $n$ is a prime, then $V_{n}$ graph consists of a single vertex. Hence it is a connected graph. In other cases, by the definition of adjacent in $V_{n}$, there exist edges between prime number vertices and their prime power vertices and also to their prime product vertices. Therefore, each vertex of $V_{n}$ is connected to some vertex in $V_{n}$.

\section{CARTESIAN PRODUCT GRAPHS}

The Cartesian product of graphs is a straight forward and natural construction. According to Imrich and Klavzar [4] Cartesian products of graphs were defined in 1912 by Whitehead and Russell [5]. These products were repeatedly rediscover later, notably by Sabidussi [6] in 1960 .

Cartesian product graphs can be recognized efficiently, in time $O(m \log n)$ for a graph with $m$ edges and $n$ vertices [7]. For more details, refer [8] and [9].

Let $G_{1}$ and $G_{2}$ be two simple graphs with their vertex sets as $V_{1}=\left\{u_{1}, u_{2}, \ldots \ldots\right\}$ and $V_{2}=\left\{v_{1}, v_{2}, \ldots \ldots\right\}$ respectively. Then the Cartesian product of these two graphs denoted by $G_{1} \square G_{2} \quad$ is defined to be a graph whose vertex set is $V_{1} \times$ $V_{2}$, where $V_{1} \times V_{2}$ is the Cartesian product of the sets $V_{1}$ and $V_{2}$ and any two distinct vertices $\left(u_{1}, v_{1}\right)$ and $\left(u_{2}, v_{2}\right)$ of $G_{1} \times G_{2}$ are adjacent if 
(i) $u_{1}=u_{2}$ and $v_{1} v_{2} \in E\left(G_{2}\right)$ or

(ii) $u_{1} u_{2} \in E\left(G_{1}\right)$ and $v_{1}=v_{2}$.

\section{RESULTS}

Let $G_{1}$ be an Euler Totient Cayley graph and $G_{2}$ be an Arithmetic $V_{n}$ graph. Then $G_{1}$ and $G_{2}$ are simple graphs as they have no loops and multiple edges. Hence by the definition of adjacency in Cartesian product, $G_{1} \square G_{2}$ is also a simple graph.

Now investigate some properties of $G_{1} \square G_{2}$.

Theorem 2.1: The degree of a vertex in the Cartesian product graph $G_{1} \square G_{2}$ is given by

$\operatorname{deg}_{G_{1} \square G_{2}}\left(u_{i}, v_{j}\right)=\operatorname{deg}_{G_{1}}\left(u_{i}\right)+\operatorname{deg}_{G_{2}}\left(v_{j}\right)$

where $u_{i} \in V_{1}$ and $v_{j} \in V_{2}$.

Proof: By the definition of Cartesian product, vertex $\left(u_{i}, v_{j}\right)$ in $G_{1} \square G_{2}$ is adjacent to all the vertices of the sets $\left\{u_{i} \times N_{G_{2}}\left(v_{j}\right)\right\}$ and $\left\{N_{G_{1}}\left(u_{i}\right) \times v_{j}\right\}$ where $N_{G_{1}}\left(u_{i}\right)$ denotes the open neighbourhood set of $u_{i}$ in the graph $G_{1}$ and $N_{G_{2}}\left(v_{j}\right)$ denotes the open neighbourhood set of $v_{j}$ in $G_{2}$.

$$
\begin{aligned}
& \text { So } N_{G_{1} \square G_{2}}\left(u_{i}, v_{j}\right)=\left\{u_{i} \times N_{G_{2}}\left(v_{j}\right)\right\} \cup\left\{N_{G_{1}}\left(u_{i}\right) \times v_{j}\right\} \\
& \text { Further }\left|N_{G_{1}}\left(u_{i}\right)\right|=\operatorname{deg}_{G_{1}}\left(u_{i}\right) \text { and } \\
& \qquad\left|N_{G_{2}}\left(v_{j}\right)\right|=\operatorname{deg}_{G_{2}}\left(v_{j}\right) . \\
& \left|N_{G_{1} \square G_{2}}\left(u_{i}, v_{j}\right)\right|=\operatorname{deg}_{G_{1} \square G_{2}}\left(u_{i}, v_{j}\right) .
\end{aligned}
$$

Now

$$
\begin{aligned}
\left|N_{G_{1} \square G_{2}}\left(u_{i}, v_{j}\right)\right| & =\left|\left\{u_{i} \times N_{G_{2}}\left(v_{j}\right)\right\}\right|+\left|\left\{N_{G_{1}}\left(u_{i}\right) \times v_{j}\right\}\right| \\
& =\operatorname{deg}_{G_{2}}\left(v_{j}\right)+\operatorname{deg}_{G_{1}}\left(u_{i}\right) .
\end{aligned}
$$

Hence $\operatorname{deg}_{G_{1} \square G_{2}}\left(u_{i}, v_{j}\right)=\operatorname{deg}_{G_{1}}\left(u_{i}\right)+\operatorname{deg}_{G_{2}}\left(v_{j}\right)$.

Remark: Since graph $G_{1}$ is a $\varphi(n)$ - regular graph, we have $\operatorname{deg}_{G_{1}}\left(u_{i}\right)=\varphi(n)$, for any $i$. Hence we can write $\operatorname{deg}\left(u_{i}, v_{j}\right)=\varphi(n)+\operatorname{deg}\left(v_{j}\right)$.

Theorem 2.2: $G_{1} \square G_{2}$ is a simple finite graph without isolated vertices.

Proof: Since $G_{1}$ and $G_{2}$ are simple finite graphs, by the definition of Cartesian product it follows that $G_{1} \square G_{2}$ is also a simple finite graph.

Since $G_{1}$ is a graph without isolated vertices for all values of $n$ $\operatorname{deg}_{G_{1}}\left(u_{i}\right) \neq 0$ for any $i . G_{2}$ is a single vertex graph if $n$ is a prime. Otherwise $G_{2}$ is graph without isolated vertices.

So $\operatorname{deg}_{G_{2}}\left(v_{j}\right)=0$ if $n$ is a prime and $\operatorname{deg}_{G_{2}}\left(v_{j}\right) \neq 0$ otherwise. Hence by Theorem 2.1, $\operatorname{deg}_{G_{1} \square G_{2}}\left(u_{i}, v_{j}\right) \neq 0$ for any $i, j$.

Thus $G_{1} \square G_{2}$ admits no isolated vertices.

Theorem 2.3: The number of vertices and edges in $G_{1} \square G_{2}$ is given respectively by
1. $\left|V\left(G_{1} \square G_{2}\right)\right|=\left|V\left(G_{1}\right)\right|\left|V\left(G_{2}\right)\right|$.
2. $\left|E\left(G_{1} \square G_{2}\right)\right|=\left|V\left(G_{1}\right)\right|\left|E\left(G_{2}\right)\right|+\left|V\left(G_{2}\right)\right|\left|E\left(G_{1}\right)\right|$

Proof: Let $p_{1}, p_{2}, p$ denote the number of vertices and $q_{1}, q_{2}, q$ denote the number of edges of graphs $G_{1}, G_{2}$ and $G_{1} \square G_{2}$ respectively. By the definition of Cartesian product, it follows that $p=p_{1} \cdot p_{2}$.

$$
\begin{aligned}
& \text { i.e., }\left|V\left(G_{1} \square G_{2}\right)\right|=\left|V\left(G_{1}\right)\right|\left|V\left(G_{2}\right)\right| \text {. } \\
& \left|E\left(G_{1}\right)\right|=q_{1}=\frac{1}{2} \sum_{i \in V_{1}} \operatorname{deg}\left(u_{i}\right) \\
& \left|E\left(G_{2}\right)\right|=q_{2}=\frac{1}{2} \sum_{j \in V_{2}} \operatorname{deg}\left(v_{j}\right)
\end{aligned}
$$

Now

$$
\begin{aligned}
\left|E\left(G_{l} \square G_{2}\right)\right|=q=\frac{1}{2} \sum_{i, j} \operatorname{deg}\left(u_{i}, v_{j}\right) \\
=\frac{1}{2}\left\{\sum_{i, j}\left[\operatorname{deg}\left(u_{i}\right)+\operatorname{deg}\left(v_{j}\right)\right]\right\} \quad \text { (By Theorem 2.1) } \\
\quad=\frac{1}{2}\left\{\left(\sum_{i, j} \operatorname{deg}\left(u_{i}\right)\right)+\left(\sum_{i, j} \operatorname{deg}\left(v_{j}\right)\right)\right\} \\
\quad=\frac{1}{2}\left\{\sum_{j}\left(\sum_{i} \operatorname{deg}\left(u_{i}\right)\right)+\sum_{i}\left(\sum_{j} \operatorname{deg}\left(v_{j}\right)\right)\right\} \\
=\frac{1}{2}\left\{\sum_{j}\left(2 q_{1}\right)+\sum_{i}\left(2 q_{2}\right)\right\} \\
=\frac{1}{2}\left\{p_{2}\left(2 q_{1}\right)+p_{1}\left(2 q_{2}\right)\right\} \\
=p_{1} q_{2}+p_{2} q_{1} \\
=\left|V\left(G_{1}\right)\right|\left|E\left(G_{2}\right)\right|+\left|V\left(G_{2}\right)\right|\left|E\left(G_{1}\right)\right| \mathbf{a}
\end{aligned}
$$

Now examine the property of connectivity in Cartesian product of these graphs.

It is proved by Wilfried Imrich and Sandi Klavzar [10] that the Cartesian product of two graphs is connected if and only if both the graphs are connected.

Since the graphs $G_{1}$ and $G_{2}$ are connected, the following result is an immediate consequence.

Theorem 2.4: $G_{1} \square G_{2}$ is a connected graph.

Theorem 2.5: $G_{1} \square G_{2}$ is a complete graph, if $n$ is a prime.

Proof: Suppose $n$ is a prime. Then Euler totient Cayley graph $G_{1}$ is a complete graph and Arithmetic $V_{n}$ graph $G_{2}$ is a single vertex graph $K_{1}$. Hence by the definition of Cartesian product, $G_{1} \square G_{2}$ becomes a complete graph

It is known that a graph is bipartite if and only if it contains no odd cycles.

To examine the property of bipartite of $G_{1} \square G_{2}$, recall the following result given by Sabidussi.

Result: A Cartesian product graph is bipartite if and only if each of its factors is bipartite.

Assume that $G_{1}$ and $G_{2}$ are bipartite. By the definition of Cartesian product, each cycle in $G_{1} \square G_{2}$ has edges either from $G_{1}$ or from $G_{2}$ (but not both). Since $G_{1}$ and $G_{2}$ are bipartite, these edges form an even cycle in $G_{1}$ or an even cycle in $G_{2}$. So the number of edges of the cycle in $G_{1} \square G_{2}$ is even. Hence there is no odd cycles in $G_{1} \square G_{2}$. Hence $G_{1} \square G_{2}$ is bipartite.

Conversely if $G_{1} \square G_{2}$ is bipartite then there are no odd cycles in $G_{1} \square G_{2}$. Since both $G_{1}$ and $G_{2}$ are subgraphs of $G_{1} \square G_{2}$, it follows that there are no odd cycles in $G_{1}$ and there are no odd cycles in $G_{2}$. Hence they are bipartite.

We now examine for what values of $n$, the Cartesian product 
$G_{1} \square G_{2}$ is a bipartite graph?

By the above result given by Sabidussi, bipartition of graph $\mathrm{G}_{1} \square \mathrm{G}_{2}$ depends on the bipartition of both the graphs $\mathrm{G}_{1}$ and $G_{2}$.

As proved by Madhavi, Euler Totient Cayley Graph $G_{1}$ is not bipartite for odd values of $n$ and it is bipartite for even values of $n$. This implies that Cartesian product graph $G_{1} \square G_{2}$ may bipartite only for even values of $n$ and not for its odd values.

Theorem 2.6: Let $n$ be an even number such that $n>2$, $n=2^{\alpha}$ or $n=2 p$ where $p$ is a prime. Then the Cartesian product graph $G_{1} \square G_{2}$ is a bipartite graph.

Proof: Suppose $n$ is an even number such that $n>2$, $n=2^{\alpha}$ or $n=2 p$ where $p$ is a prime. Then Euler totient Cayley graph $G_{1}$ is a bipartite graph.

Now it has to be proved that $G_{2}$ is also a bipartite graph by showing that $G_{2}$ contains no odd cycles. The proof follows in two cases.

Case 1: Suppose $n=2^{\alpha}$.

In this case Arithmetic graph $G_{2}$ contains the vertices $2,2^{2}, 2^{3}, \ldots \ldots \ldots, 2^{\alpha}$. Since $\operatorname{GCD}\left(2^{i}, 2^{j}\right) \neq 2$ for any $i, j>1$, there exists no edge between any two powers of 2 . The only edges are between 2 and its powers. Hence odd cycles cannot occur in $G_{2}$.

Case 2: Suppose $n=2 p$ where $p$ is a prime.

In this case Arithmetic graph $G_{2}$ has the vertices $2, p$ and $2 p$. Then by the definition of edges in $G_{2}$, there are edges between 2 and $2 p$ since $\operatorname{GCD}(2,2 p)=2$ and $p$ and $2 p$ since $\operatorname{GCD}(p, 2 p)=p$. Since $p$ being an odd prime, we have $\operatorname{GCD}(2, p)=1$. This implies that there is no edge between the vertices 2 and $p$ of $G_{2}$. Thus $G_{2}$ has no odd cycle.

Thus in either of the cases, $G_{2}$ has no odd cycle. And hence it is a bipartite graph. Therefore $G_{1}$ and $G_{2}$ are bipartite graphs if the even number $n>2$ is of the form $2^{\alpha}$ or $2 p$ which implies that the Cartesian product graph $G_{1} \square G_{2}$ is a bipartite graph.

Theorem 2.7: $G_{1} \square G_{2}$ is not a bipartite graph, if the even number $n$ is neither in the form $2^{\alpha}$ nor $2 p$.

Proof: Suppose $n$ is an even number such that $n \neq 2^{\alpha}$ or $n \neq 2 p$ where $p$ is a prime.

Since $n$ being an even number, Euler totient Cayley graph $G_{1}$ is a bipartite graph. Since the even number $n$ is not in the form $2^{\alpha}$ and $2 p$, it can be written as

$n=2^{\alpha} p_{1}^{\alpha_{1}} p_{2}^{\alpha_{2}} \ldots \ldots . . p_{k}^{\alpha_{k}}$, where $p_{1}, p_{2}, \ldots, p_{k}$ are odd primes and $\alpha_{i} \geq 1$. Then $G_{2}$ contains three distinct vertices $2,2 p_{i}, 2 p_{j}$ with $\operatorname{GCD}\left(2,2 p_{i}\right)=2, \quad \operatorname{GCD}\left(2,2 p_{j}\right)=2$, and GCD $\left(2 p_{i}, 2 p_{j}\right)=2$. This implies that these vertices are connected by edges. So, $G_{2}$ contains an odd cycle and hence it is not bipartite.

Now, $G_{1}$ is a bipartite graph and $G_{2}$ is not a bipartite graph implies that $G_{1} \square G_{2}$ is not a bipartite graph.

\section{ILLUSTRATIONS}

Let $n=4$.

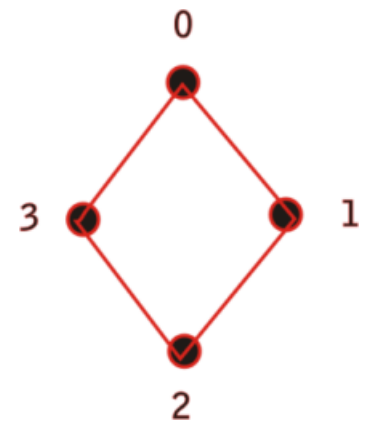

Fig 1

$$
G_{1}=G\left(Z_{4}, \varphi\right)
$$

Fig 2

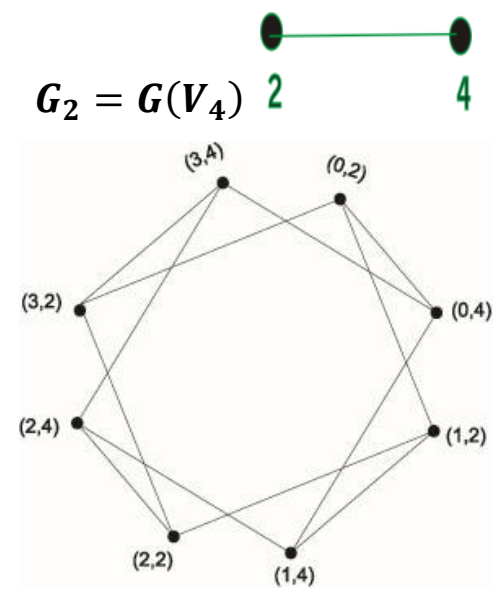

Fig 3

$\boldsymbol{G}_{\mathbf{1}} \square \boldsymbol{G}_{\mathbf{2}}$

Let $\boldsymbol{n}=\mathbf{6}$

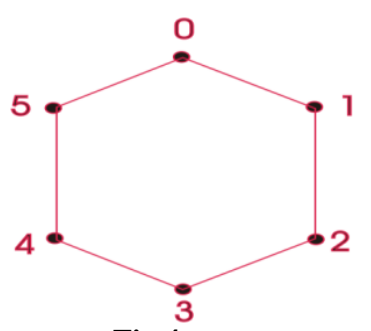

Fig 4

$G_{1}=G\left(Z_{6}, \varphi\right)$

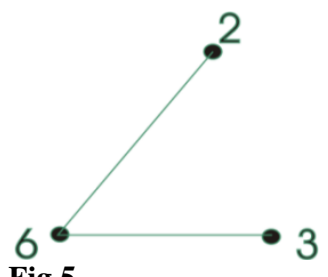

Fig 5

$G_{2}=G\left(V_{6}\right)$ 


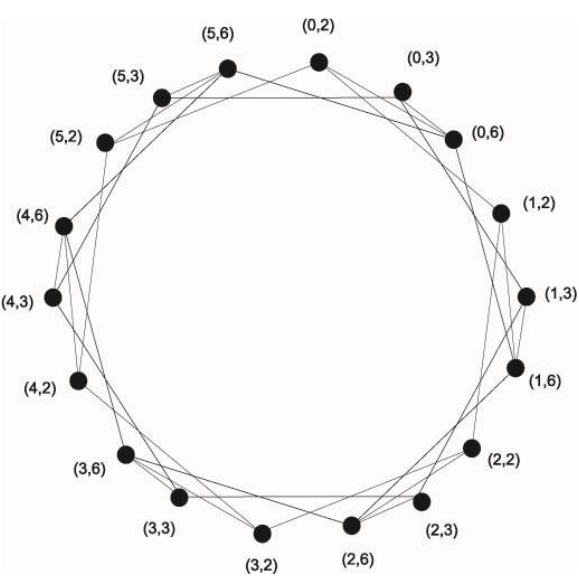

Fig 6, $G_{1} \square G_{2}$

Let $n=\mathbf{1 1}$

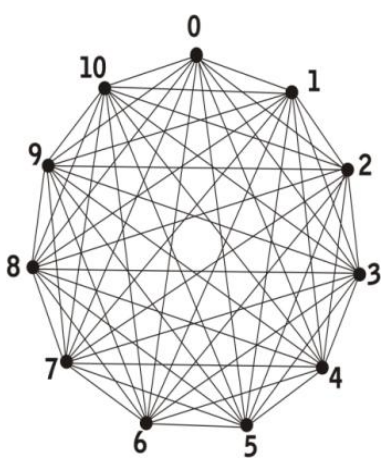

Fig 7

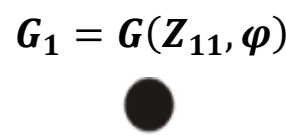

Fig 8

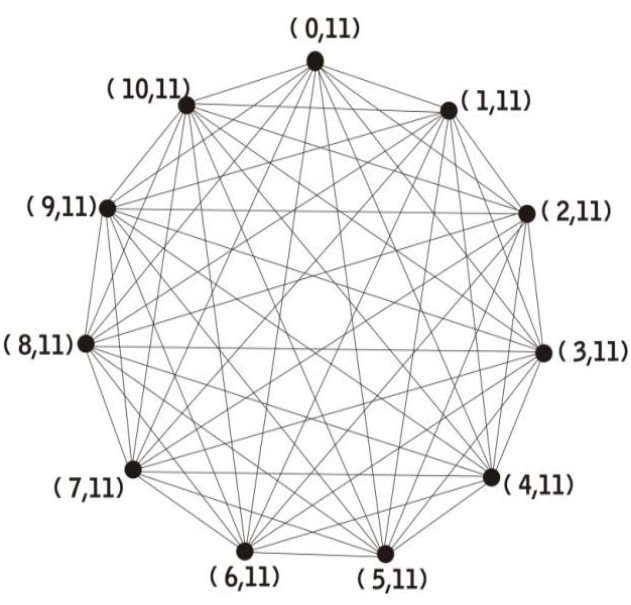

$G_{2}=G\left(V_{11}\right)$

Fig $9, G_{1} \square G_{2}$

\section{CONCLUSION}

Graph Theory is young but rapidly maturing subject. Its basic concepts are simple and can be used to express problems from many different subjects. The purpose of this work is to familiarize the reader with the Cartesian product graph of Euler Totient Cayley graph with Arithmetic $\mathrm{V}_{\mathrm{n}}$ graph.

It is useful other Researchers for further studies of other properties of these product graphs and their relevance in both combinatorial problems and classical algebraic problems.

\section{ACKNOWLEDGMENTS}

This work was supported by the University Grants Commissions with Grant No.

\section{F MRP-5510 /15 (SERO/UGC)}

\section{REFERENCES}

[1] Madhavi, L.-Studies on domination parameters and enumeration of cycles in some Arithmetic Graphs, Ph.D Thesis submitted to S.V.University, Tirupati, India, (2002).

[2] Sujatha, K.- Studies on domination parameters and cycle structures of Cayley graphs associated with some arithmetic functions. Ph. D. Thesis submitted to S.V. University, Tirupati, India, (2008).

[3] Vasumathi, N. - Number theoretic graphs, Ph. D. Thesis submitted to S.V.University, Tirupati, India, (1994).

[4] Imrich, W. and Klavzar, S.- Product Graphs: Structure and Recognition. John, Wiley \& Sons, New York, USA (2000)

[5] Whitehead, A.N. and Russel, B. Principia Mathematica, Volume 2, Cambridge, University Press, Cambridge (1912).

[6] Sabidussi, G. - Graph multiplication, Mathematics chef Festschrift 72: 446-457, (1960)

[7] Amrich Wilfried \& Peterin Iztok. -Recognizing Cartesian products in linear time, Discrete Mathematics 307 (3-5): $472-483$ (2007).

[8] Vizing, V.G. The Cartesian product of graphs, Comp. El Syst. 2, 352-365 (1963)

[9] Harary, F- On the group of the composition of two graphs - Duke Math. J., 26, 29-36 (1959)

[10] Imrich, W., Klavžar, S. \& Rall, Douglas F.- Graphs and their Cartesian Products, A. K. Peters, ISBN 1-56881429-1(2008)

[11] L.R. Foulds - Graph Theory Applications, Springer Verlag, New York, 17-25 (1992)

[12] Bela Bollobas - Modern Graph Theory -Springer International Edition (2013)

[13] Kenneth H Rosen. -Discrete Mathematics and its Applications-with Combinatorics and Graph TheorySeventh Edition (2014). 